\title{
How safe is taxis in hernia? Reduction of gangrenous omentum - an uncommon occurrence
}

\author{
Gaurav Garg ${ }^{1}$, Ratnesh Jenaw ${ }^{2}$, Siddharth Chaudhary ${ }^{3}$, K. M Garg ${ }^{4}$
}

\begin{abstract}
Use of taxis (hernia reduction) subsided in favour of urgent herniorrhaphy with advent of safer general anaesthesia. Although, the danger of taxis seem to be rare, but it resulted in reduction of gangrenous omentum in our case. So taxis is not safe in modern era as signs of strangulation are not always obvious on clinical examination.
\end{abstract}

Key words: Hernia, Incarceration, taxis.

\section{Introduction}

Since the first attempt of hernia reduction in the $4^{\text {th }}$ century BC by the Greek Praxagoras of Cas, taxis was the standard and only treatment to save the patient's life. Although the first emergency herniorrhaphy for incarcerated hernia was performed in the $16^{\text {th }}$ century by Maupasius and Pierre Franco, it was not until the discovery of general anaesthesia in the mid- $19^{\text {th }}$ century that emergency repair replaced taxis as a treatment of choice $^{1}$. However, the role of taxis in current hernia treatment continues to be debated. Herein we report a case in whom by taxis reduction of gangrenous omentum occurred.

\section{Case Report}

A 30 years old male presented to MGMCH, Jaipur Surgery out patient department with history of reducible right inguinal swelling of one month duration, but for last one day swelling was painful and irreducible having size of $15 \mathrm{~cm} \times 10 \mathrm{~cm}$. Taxis was attempted and successful. Patient was advised surgery the next day but he refused so and went home.

He came back a week after with full blown picture of strangulated right inguinal hernia with swelling of $5 \mathrm{~cm} \times 10 \mathrm{~cm}$., tender, raised local temperature, firm, nonreducible, absent cough impulse, pulse rate 104/min, vomiting, evidences of dehydration and absent bowel sounds. Laboratory investigations revealed total leucocyte count as $13200 / \mathrm{cmm}$, blood urea $67 \mathrm{mg} / \mathrm{dl}$, S. Creatinine $0.9 \mathrm{mg} / \mathrm{dl}$, upright plain abdominal X-ray showed multiple air fluid levels.

After resuscitation emergency surgery was done. On right inguinal exploration about $100 \mathrm{ml}$ blackish colored fluid was present inside hernia sac with gangrenous omentum. On separation of adherent omentum near neck, when finger entered in peritoneal cavity a hard mass was felt in the lower abdomen. So a formal Laparotomy with lower midline incision was performed and to our surprise whole of the omentum was gangrenous, which was resected. Patient had an uneventful post operative recovery. He was well when last seen at 6 months follow up.

\section{Comments}

The role of taxis (Hernia reduction) in current hernia treatment continues to be debated. An attempt at reduction is reasonable unless there are signs of strangulation, which is not always obvious by clinical examination ${ }^{2}$. Overall the traditionally evoked danger of taxis seem to be very rare and include-contusion or rupture of intestinal wall, reduction en masse, reduction into the loculus of the sac and rupture of sac leading to reduction of contents extraperitoneally ${ }^{3}$. However, there is no calculated risk for such an event reported in the available medical literature.

Recently, supporters of taxis ${ }^{1}$ in their experience of sixty-one incarcerated hernias reported no taxis related complications during follow up and they have concluded that taxis can lower morbidity and mortality in patients who, otherwise would have undergone an emergency surgery. Similarly, others ${ }^{4}$ support taxis in elderly patients as it can delay surgery until other medical problems are optimally controlled as well as it provides better local conditions by allowing the edema and inflammation to stabilize and, as a result the operative procedure become less traumatic. However, these authors ${ }^{1,4}$ have suggested the need for controlled randomized studies for optimal premedication before attempting taxis.

The present patient reported with only one day history of pain and irreducibility, taxis was attempted in out patient department and was successful. but patient came back a week after with strangulated right inguinal hernia and on operation it was gangrenous omentocele. On laparotomy the omentum inside the peritoneal cavity was also gangrenous, suggesting that it was the result of initial taxis attempt leading to reduction of gangrenous omentum. 
The main concern in taxis is the quantum of force applied. Further, it is also not known that how many clinicians have seen the patient before?. Every clinician attempts taxis and there is always possibility of additive effect of force applied. Though it has been warned that the maneuver of taxis should not be performed with excessive pressure ${ }^{2}$. Vigorous manipulation has no place in modern era and is mentioned only to be condemned $^{3}$.

In incarcerated hernia, immediate surgical exploration is the safest approach when the diagnosis is not clear ${ }^{2}$. Though strangulation is usually clinically evident, gangrenous tissue can occasionally be reduced into the abdomen by manual or spontaneous reduction. It is therefore safest to repair the reduced hernia at the earliest opportunity. At surgery, one must decide whether to explore the abdomen to make certain that the intestine is viable. If patient has leucocytosis or clinical signs of peritonitis or if the hernia sac contains dark or bloody fluid, the abdomen should be explored ${ }^{5}$. The risks of attending an operation for strangulated hernia are very much exaggerated, however fatality for these cases is not due to the operation, but to the hopeless condition of the contents of the sac, too often consequence of delay prior to the relief which an operation is designed to afford.

In our opinion putting the patient in trendelenburg with foot end of bed resting on wooden blocks, flexing the thigh, putting an icecap over hernia to keep down edema and giving patient sedation will reduce most incarcerated hernia by a gentle gravity pull from the inside. This age old treatment is still the safest and best. Any hernia which is not so reduced should certainly not be manipulated, lest traditionally evoked danger of taxis may occur.

Hence, taxis is not safe in modern era as signs of strangulation are not always obvious by clinical examination and further we recommend deletion of word "taxis" from modern surgical teaching.

\section{Bibliography}

[1]. Harissis H.V, E. Douitsis, M. Fatouros Incarcerated hernia: to reduce or not to reduce? Hernia (2009)13:263-266 DOI 10.1007/s10029-008-0467-9

[2]. Greenfield Surgery-Scientific Principles and practice. Fifth edition, Vol. 1,2011, Lippincott Williams and Wilkins, a Wotters Kluwer business, Philadelphia, USA

[3]. Bailey and Loves: Short practice of Surgery, 25 ${ }^{\text {th }}$ edition,2008. Published by Hodder Arnold, an imprint of Hodder education, an Hachette UK company; 338 Euston Road, London NW13BH.

[4]. Rosenthal RA, Andersen DK (1998) Physiologic consideration in the elderly surgical patients. In: Miller TA (ed.) Modern surgical care, vol 2. Quality Medical Publishing Inc., St. Louis, pp 1362-1384.

[5]. Current diagnosis and treatment surgery, thirteenth edition, edited by Gerard M. Doherty Mc Grew Hill Companies Inc Printed in USA. 